\title{
Status of and prospects for advanced tokamak regimes from multi-machine comparisons using the 'International Tokamak Physics Activity' database
}

\author{
X Litaudon ${ }^{1,23}$, E Barbato ${ }^{2}$, A Bécoulet ${ }^{1}$, E J Doyle ${ }^{3}$, T Fujita ${ }^{4}$, P Gohil ${ }^{5}$, \\ F Imbeaux ${ }^{1}$, O Sauter ${ }^{6}$, G Sips ${ }^{7}$, for the International Tokamak Physics \\ Activity (ITPA) Group on Transport and Internal Transport Barrier \\ (ITB) Physics: J W Connor ${ }^{8}$, E J Doyle ${ }^{3}$, Yu Esipchuk ${ }^{9}$, T Fujita ${ }^{4}$, \\ T Fukuda ${ }^{10}$, P Gohil ${ }^{5}$, J Kinsey ${ }^{11}$, N Kirneva ${ }^{9}$, S Lebedev ${ }^{12}$, X Litaudon ${ }^{1}$, \\ V Mukhovatov ${ }^{13}$, J Rice ${ }^{14}$, E Synakowski ${ }^{15}$, K Toi ${ }^{16}$, B Unterberg ${ }^{17}$, \\ V Vershkov ${ }^{9}$, M Wakatani ${ }^{18,24}$ and for the International ITB Database \\ Working Group and the responsible officers for the ITPA collaborative \\ experiments on the 'hybrid' and 'steady-state' regimes: T Aniel',

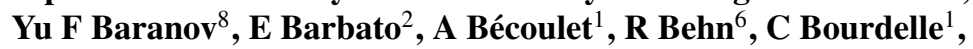 \\ G Bracco ${ }^{2}$, R V Budny ${ }^{15}$, P Buratti ${ }^{2}$, E J Doyle ${ }^{3}$, Yu Esipchuk ${ }^{9}$, \\ B Esposito ${ }^{2}$, S Ide ${ }^{4}$, A R Field ${ }^{8}$, T Fujita ${ }^{4}$, T Fukuda ${ }^{4}$, P Gohil $^{5}$, \\ C Gormezano $^{2}$, C Greenfield ${ }^{5}$, M Greenwald ${ }^{14}$, T S Hahm ${ }^{15}$, \\ G T Hoang ${ }^{1}$, J Hobirk ${ }^{7}$, D Hogeweij ${ }^{19}$, S Ide ${ }^{4}$, A Isayama ${ }^{4}$, F Imbeaux ${ }^{1}$, \\ E Joffrin ${ }^{1}$, Y Kamada ${ }^{4}$, J Kinsey ${ }^{10}$, N Kirneva ${ }^{9}$, X Litaudon ${ }^{1}$, T C Luce ${ }^{5}$, \\ M Murakami $^{20}$, V Parail $^{8}$, Y-K M Peng ${ }^{20}$, F Ryter $^{7}$, Y Sakamoto ${ }^{4}$, \\ H Shirai $^{4}$, G Sips ${ }^{7}$, T Suzuki ${ }^{4}$, E Synakowski ${ }^{15}$, H Takenaga ${ }^{4}$, \\ T Takizuka ${ }^{4}$, T Tala ${ }^{21}$, M R Wade ${ }^{20}$ and J Weiland ${ }^{22}$ \\ ${ }^{1}$ Association EURATOM-CEA, 13108 St Paul lez Durance, France \\ 2 Associazione EURATOM-ENEA sulla Fusione, C.R. Frascati, Frascati, Italy \\ ${ }^{3}$ University of California, Los Angeles, CA 90095, USA \\ ${ }^{4}$ JAERI, Naka Fusion Research Establishment, Naka, Japan \\ 5 General Atomics, PO Box 85608, San Diego, CA 92186-5608, USA \\ ${ }^{6}$ Association Euratom-Confédération Suisse, CRPP, CH-1015 Lausanne, Switzerland \\ ${ }^{7}$ Max-Planck-Institut für Plasmaphysik, EURATOM Association, Garching, Germany \\ ${ }^{8}$ UKAEA-EURATOM Association, Culham Science Centre, Abingdon, OX14 3DB, UK \\ ${ }^{9}$ Kurchatov Institute of Atomic Energy, Moscow, Russia \\ ${ }^{10}$ Osaka University, Suita, Japan \\ ${ }^{11}$ Lehigh University, Bethlehem, PA 18015, USA \\ 12 Ioffe Institute, St Petersburg, Russia \\ 13 ITER JWS, Naka, Japan \\ ${ }^{14}$ Massachusetts Institute of Technology, Cambridge, MA 02139, USA \\ 15 Plasma Physics Laboratory, Princeton University, Princeton, NJ 08543, USA \\ 16 National Institute of Fusion Science, Toki City, Japan \\ ${ }^{17}$ Institut für Plasmaphysik, Forschungszentrum Jülich GmbH Association EURATOM-FZJ, \\ Jülich, Germany \\ 18 Kyoto University, Kyoto, Japan \\ 19 FOM Insituut voor Plasmafisica, 'Rijnhuizen', Nieuwegein, The Netherlands \\ ${ }^{20}$ Oak Ridge National Laboratory, Oak Ridge, TN 37831, USA
}

\footnotetext{
${ }^{23}$ Author to whom any correspondence should be addressed.

${ }^{24}$ Deceased.
} 


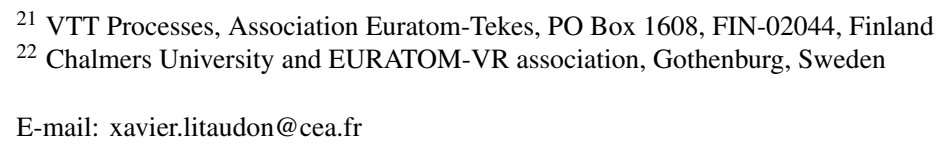

Received 21 November 2003

Published 5 April 2004

Online at stacks.iop.org/PPCF/46/A19

DOI: $10.1088 / 0741-3335 / 46 / 5 \mathrm{~A} / 002$

\begin{abstract}
Advanced tokamak regimes obtained in ASDEX Upgrade, DIII-D, FT-U, JET, JT-60U, TCV and Tore Supra experiments are assessed both in terms of their fusion performance and capability for ultimately reaching steady-state using data from the international internal transport barrier database. These advanced modes of tokamak operation are characterized by an improved core confinement and a modified current profile compared to the relaxed Ohmically driven one. The present results obtained in these experiments are studied in view of their prospect for achieving either long pulses ('hybrid' scenario with inductive and non-inductive current drive) or ultimately steady-state purely non-inductive current drive operation in next step devices such as ITER. A new operational diagram for advanced tokamak operation is proposed where the figure of merit characterizing the fusion performances and confinement, $H \times \beta_{\mathrm{N}} / q_{95}^{2}$, is drawn versus the fraction of the plasma current driven by the bootstrap effect. In this diagram, present day advanced tokamak regimes have now reached an operational domain that is required in the non-inductive ITER current drive operation with typically $50 \%$ of the plasma current driven by the bootstrap effect (Green et al 2003 Plasma Phys. Control. Fusion 45 587). In addition, the existence domain of the advanced mode regimes is also mapped in terms of dimensionless plasmas physics quantities such as normalized Larmor radius, normalized collisionality, Mach number and ratio of ion to electron temperature. The gap between present day and future advanced tokamak experiments is quantitatively assessed in terms of these dimensionless parameters.
\end{abstract}

\title{
1. Introduction
}

High thermonuclear fusion yield operating scenario foreseen in the next step tokamaks such as ITER relies mainly on the plasmas performances presently achieved in inductive current drive regime with an edge transport barrier (H-mode) for reducing the anomalous radial transport (Campbell et al 2001). This mode of operation, for which an extensive experimental database exists, is attractive for reaching high fusion power but is not foreseen to achieve a genuine steady-state where the plasma current is solely driven by non-inductive current drive means. In the last 10 years, much effort and progress has been made by developing regimes that could lead to efficient and purely non-inductive current drive tokamak operation with a large fraction of the plasma current self-generated (pressure driven) by the neoclassical 'bootstrap' effect (e.g. Kikuchi (1990)). The performance of these non-inductive regimes is usually achieved by tailoring or controlling the shape of the current density profile leading to the formation of a core region with reduced anomalous radial transport (i.e. with improved core confinement) that could take the form (but not necessarily) of an internal transport barrier (ITB). In this 
context, ITER is designed for both inductive and purely non-inductive current drive operation (Campbell et al 2001, Green et al 2003). In the inductive mode that solely relies on the edge transport barrier for improving the global plasma confinement properties, the safety factor profile ( $q$-profile) has a standard monotonic Ohmic shape with a minimum value, $q_{\mathrm{min}}$, located on-axis that stays below unity. The expected performance is an improved confinement factor over standard L-mode prediction, $H_{\mathrm{L}}$, of the order of 2 with a normalized toroidal beta, $\beta_{\mathrm{N}} \sim 2$, at high plasma current and low $q$ at the plasma surface $\left(H_{\mathrm{L}} \times \beta_{\mathrm{N}} \sim 4\right.$ at $\left.q_{95} \sim 3\right)$. On the contrary, steady-state operation performed at lower plasma current $\left(q_{95} \sim 5\right)$ should be made compatible with a larger fraction of off-axis bootstrap current (typically above 50\%). The rest of the current is driven with external non-inductive current drive means to reach full noninductive current drive operation. In this case, operation with non-monotonic $q$-profile (zero or negative magnetic shear in the core) is envisaged with typically $q_{\min }$ lying between 1.5 and 2.5. To compensate for the reduction of plasma confinement linked to a lower plasma current for optimizing the bootstrap current fraction, steady-state operation requires to further improve the confinement compared to the edge transport barrier with typically $H_{\mathrm{L}}>3$ at $\beta_{\mathrm{N}}>3$ : $H_{\mathrm{L}} \times \beta_{\mathrm{N}} \sim 9$ at $q_{95} \sim 4-5$ is expected for this regime in ITER with 50\% of bootstrap current. It should be pointed out that in an economical steady-state thermonuclear tokamak reactor, the bootstrap current should be further optimized $(\sim 80 \%)$ to reduce the need for external non-inductive current drive sources. Finally, an intermediate step between these two extreme modes of tokamak operation is generally referred as the 'hybrid' scenario in which a large (but not the whole) fraction of the plasma current is non-inductively driven. Therefore, in this intermediate case the $q$-profile is monotonic and stays slightly above unity with a weak magnetic shear in the core. Fusion performance in the range of $H_{\mathrm{L}} \times \beta_{\mathrm{N}} \sim 5$ at $q_{95} \sim 3-4$ is expected for this regime in ITER. With a total non-inductive current fraction of the order of $50 \%$, the 'hybrid scenario' is envisaged to extend the pulse duration of the standard inductive $\mathrm{H}$-mode regime.

If the inductive $\mathrm{H}$-mode is now relatively well explored, an open issue is how the presently developed non-inductive current drive regimes ('hybrid and 'steady-state' regimes that refer as 'advanced tokamak' regimes thereafter in this paper) will extrapolate to next step thermonuclear burning plasmas experiments such as ITER. In this context, this paper will focus on the status and prospect of advanced tokamak regimes for future tokamak operation using multi-machine data from the international ITB database. The inter-machine database consists of both zero- and one-dimensional (radial profile) data obtained from many devices: ASDEX Upgrade, DIII-D, FT-U, JET, JT-60U, RTP, T10, TCV and Tore Supra (Fukuda et al 2001). The international ITB database is constructed to address issues regarding the conditions required to form an ITB, the core confinement properties, and the assessment of the various transport models with plasma profiles from different machines. The international ITB database was initially set up by Fukuda et al (2001) and the first studies on the power threshold scaling to trigger an ion or electron ITB were carried out by Sips et al (2002a). The crucial role played by the safety factor profile and the low magnetic shear to reduce the power requirement for ITB formation has been studied by Hoang et al (2002). Using data from the profile multi-machine database, Gohil et al (2003) have assessed various predictive transport models by comparing a pair of discharges from DIII-D, JET and JT-60U with, respectively, monotonic or non-monotonic safety factor profiles. More recently, Fujita et al (2003) have investigated the critical values of the temperature scale length for the ITB formation in many devices also using the multi-machine profile database. In this context, briefly summarized in this introduction, the international ITB database has been mainly used to study the ITB formation conditions. For the work described in this paper, new entries have been added to the international database that consist of data taken during the well developed high performance phase discharges representing either 
the 'hybrid' or the steady-state scenarios as foreseen in the ITER non-inductive current drive operation.

After this introduction, the paper is divided in three main parts. In section 2, the devices, the set of experiments and the data that have been selected for this paper are presented in detail. Then, section 3 is devoted to the assessment of present day advanced tokamak experiments with common figures of merit using data from the multi-machine database. The presently achieved plasma fusion performances are analysed in view of their prospect for long pulse ('hybrid' regime) or fully non-inductive current drive steady-state operation. The progress of advanced tokamak regimes is assessed in a diagram where the figures of merit of the fusion performances are represented versus their capability for steady-state. The presently explored operational domain is systematically compared to the required range of plasma parameters expected in next step experiments such as ITER. The operational domains of the various devices are also mapped in terms of dimensionless plasmas parameters such as the normalized Larmor radius, normalized collisionality, Mach number, and the ratio of ion to electron temperature. Finally, in section 4 a conclusion is proposed on the basis of the reported analysis by highlighting unexplored or the not so well explored plasma domains where experimental efforts could be made to reduce the uncertainties when extrapolating the present day advanced regimes to next step experiments.

\section{Description of the global database for advanced tokamak regimes}

To assess the plasma performances of the various advanced regimes obtained in present day tokamaks experiments, the international zero-dimensional ITB database has been recently updated in September 2003 with data taken during the well developed high performance phase of discharges with improved core confinement. Indeed, previous studies using the multimachine ITB zero-dimensional database were more focused on the definition of a scaling law for the power threshold to trigger an ITB in either the electron or ion channels (e.g. Fukuda et al (2001), Sips et al (2002a)). For this research activity, the data previously used were selected prior to or just at the ITB onset time, i.e. in the prelude phase of the scenario with reduced fusion performance. On the contrary, for the analyses reported in this paper, the values of the plasma parameters have been taken during the high fusion performance phase of the corresponding discharge: at the time when the neutron yield reaches its maximum value. Only one time slice has been selected for each discharge. In addition, we have included in the international ITB database not only the transient high performance, high current ITB discharges but also the non-inductive current drive regimes, i.e. both the 'hybrid' and the steady-state scenarios. As discussed in the introduction, the 'hybrid' regime is considered as an intermediate step between the standard inductive H-mode of operation and the purely noninductive current drive scenario. Improved core confinement with an ITB and neoclassical core transport is not necessarily obtained in the 'hybrid' regime characterized by a $q$-profile that is flat and slightly above unity in the plasma centre to avoid the sawtooth activity. Therefore, the database used for the present analysis encompasses a larger range of advanced tokamak regimes than previously selected for studying the conditions for ITB formation. This choice to include in the international database data from both the 'hybrid' and the highly non-inductive current drive ('steady-state') regimes is motivated by the fact that these operation modes are foreseen as two promising potential 'advanced' scenarios for the next step tokamak device such as ITER (Campbell et al 2001, Green et al 2003).

Global plasmas performances of the advanced modes of operation have been selected from experiments carried out in ASDEX Upgrade, DIII-D, FT-U, JET, JT-60U, TCV and Tore supra. For each of these tokamaks, table 1 provides an overview of the number of discharges and 
Table 1. Overview of the number of entries and regimes selected for this analysis.

\begin{tabular}{|c|c|c|c|}
\hline Tokamak & Entries & $\begin{array}{l}\text { Advanced regimes and } \\
\text { corresponding entries }\end{array}$ & References \\
\hline ASDEX Upgrade & 372 & $\begin{array}{l}\text { Ion and/or electron ITB (94) } \\
\text { 'Hybrid' regime (218) }\end{array}$ & $\begin{array}{l}\text { Wolf et al } 1999 \\
\text { Wolf et al } 2001 \\
\text { Sips et al 2002b }\end{array}$ \\
\hline FT-U & 3 & Electron ITB (3) & Pericoli-Ridolfini et al 2003 \\
\hline DIII-D & 9 & $\begin{array}{l}\text { ITB with weak and reversed magnetic shear (4) } \\
\text { Quiescent double barrier (2) } \\
\text { 'Steady-state' scenario (2) } \\
\text { 'Hybrid' scenario (1) }\end{array}$ & $\begin{array}{l}\text { Strait et al } 1995 \\
\text { Wade et al } 2003 \\
\text { Doyle et al } 2001 \\
\text { Luce } \text { et al } 2003\end{array}$ \\
\hline $\begin{array}{l}\text { JET Undertaking } \\
\text { EFDA-JET }\end{array}$ & $\begin{array}{r}25 \\
277\end{array}$ & $\begin{array}{l}\text { ITB regime with weak and reversed } \\
\text { magnetic shear } \\
\text { 'Hybrid' scenario (27) }\end{array}$ & $\begin{array}{l}\text { JET team } 1999 \\
\text { Gormezano } 1999 \\
\text { Challis et al } 2001 \text { and } 2002 \\
\text { Crisanti et al } 2002 \\
\text { Litaudon et al } 2003 \\
\text { Joffrin } \text { et al } 2002 \\
\text { Sips } \text { et al } 2003\end{array}$ \\
\hline JT-60U & 17 & $\begin{array}{l}\text { High } \beta_{\mathrm{p}} \text { regime }(9) \\
\text { Negative magnetic shear }(8)\end{array}$ & $\begin{array}{l}\text { Kamada et al } 2001 \\
\text { Ide } \text { et al } 2002 \\
\text { Fujita et al } 2001 \\
\text { Fujita } \text { et al } 2002 \mathrm{a}, \mathrm{b}\end{array}$ \\
\hline $\mathrm{TCV}$ & 10 & $\begin{array}{l}\text { Electron ITB } \\
\text { Full current drive operation } \\
\text { High bootstrap regime }\end{array}$ & $\begin{array}{l}\text { Sauter et al } 2002 \\
\text { Henderson } \text { et al } 2003 \\
\text { Behn } \text { et al } 2003\end{array}$ \\
\hline Tore Supra & 40 & $\begin{array}{l}\text { Full current drive operation (22) } \\
\text { Electron ITB (4) } \\
\text { High } \beta_{\mathrm{p}} \text {, fast wave electron heating (13) } \\
\text { Pellet enhanced mode (1) }\end{array}$ & $\begin{array}{l}\text { Jacquinot et al } 2002 \\
\text { Litaudon } \text { et al } 2001 \\
\text { Equipe TS } 1996 \\
\text { Saoutic } \text { et al } 1994 \\
\text { Géraud } \text { et al } 1994\end{array}$ \\
\hline
\end{tabular}

the regimes that have been selected together with the corresponding references. In addition to these specific references, it is worth noting that a description of the advanced tokamak mode of operation has been reviewed by Taylor (1997), Litaudon (1998), Gormezano (1999), Gohil et al (2002), Wolf (2003), Connor et al (2004) and Bécoulet et al (2003) for the JET and Tore Supra experiments. In addition, it is worth pointing out that criteria have not been given on the number of pulses to be provided to the ITPA database by each data provider of the various tokamaks. For a given device, a reduced number of entries to the ITPA database mean that only the highest fusion performance discharges of each regime have been selected and these data will appear clearly in the graphs shown in the following sections. On the contrary, when a large number of entries have been provided to the ITPA database, it means that both the highest performance discharges and the preparatory discharges to reach these high performance plasmas have been selected. In this case, these entries will also provide an indication on the route towards the high fusion performance regimes.

For each device, the data selected at the time where maximum plasma performance is reached consist of the measurements characterizing:

(i) the standard magnetic configuration ( $R$, the major radius; $a$, the minor radius; $I_{\mathrm{p}}$, the plasma current; $B_{0}$, the toroidal field on axis; $q_{95}$, the safety factor at $95 \%$ of the poloidal flux; $\kappa$, the elongation and $\delta$, the triangularity);

(ii) the global confinement time from the diamagnetic energy, $\tau_{E}$; 
(iii) the plasmas profiles (the core and volume averaged electron temperature, $T_{\mathrm{eo}}$ and $\left\langle T_{\mathrm{e}}\right\rangle$; the central, the line and volume averaged electron density, $n_{\mathrm{eo}}, n_{\mathrm{el}}$ and $\left\langle n_{\mathrm{e}}\right\rangle$; the central main ion density, $n_{\text {io }}$, the central ion temperature, $T_{\text {io }}$, and the toroidal rotation, $v_{\Phi 0}$ of the carbon impurity).

To assess the prospect of the regimes for long pulse operation, information on the duration of the high performance phase, $\tau_{\mathrm{D}}$ has been also included in the database. A common definition has been agreed among all experiments in the various devices: $\tau_{\mathrm{D}}$ is defined as the duration where the plasma performance is maintained above $85 \%$ of the maximum stored energy. This definition provides in most cases an accurate characterization of the duration of the high performance phase: excluding the duration of either the prelude phase of the scenario or any lower plasma performance phases after a back transition (MHD event, radiative collapses, etc). In addition, the following normalized quantities have been calculated:

(i) the normalized plasma pressure; the poloidal and toroidal beta are, respectively, defined as $\beta_{\mathrm{p}}=2 \mu_{0}\langle p\rangle / B_{\mathrm{p}}^{2}$ and $\beta_{\mathrm{t}}=2 \mu_{0}\langle p\rangle / B_{0}^{2}$ where $\langle p\rangle$ is the volume averaged total (thermal and non-thermal) plasma pressure and $B_{\mathrm{p}}$ is the averaged poloidal magnetic field on the last closed magnetic flux surface;

(ii) the normalized toroidal beta defined as $\beta_{\mathrm{N}}=\beta_{\mathrm{t}} \cdot\left(I_{\mathrm{p}} / a B_{0}\right)^{-1}$

(iii) the (thermal and non-thermal) confinement time normalized to the non-thermal L-mode confinement time predicted by the ITER-89P scaling law, $H_{\text {ITER-89P (revised by Kaye } \text { et al }}$ (1997));

(iv) the normalized ion Larmor radius at the sound speed $\rho^{*}=\rho_{\mathrm{s}} / a$ calculated with the volume averaged electron temperature $\left(\rho_{\mathrm{s}}=c_{\mathrm{s}} / \omega_{\mathrm{ci}} \propto T_{\mathrm{e}}^{1 / 2} m_{\mathrm{i}}^{1 / 2} /\left(Z B_{0}\right)\right.$, where $c_{\mathrm{s}}$ is the ion sound speed and $\omega_{\mathrm{ci}}$ the ion cyclotron frequency);

(v) the normalized collisionality, $v_{\mathrm{e}}^{*}$, calculated with the volume averaged electron density and temperature ( $v_{\mathrm{e}}^{*}$ is the effective collision frequency for the trapped particles normalized to their bounce frequency, $v_{\mathrm{e}}^{*} \propto n_{\mathrm{e}} q R /\left(\varepsilon^{3 / 2} T_{\mathrm{e}}^{2}\right)$ where $\left.\varepsilon=a / R\right)$;

(vi) the normalized plasma toroidal rotation Mach number $M_{\Phi}, M_{\Phi}=V_{\Phi 0} / c_{\mathrm{s}}$.

The evaluation of the non-dimensional quantities as $\beta_{\mathrm{t}}, \rho^{*}, v_{\mathrm{e}}^{*}, M_{\Phi}, T_{\mathrm{i}} / T_{\mathrm{e}}$ is crucial for comparing and extrapolating the present high performance regimes since they determine the behaviour of the key physics processes, such as stability, turbulent transport and neoclassical quantities (bootstrap, etc). The definition of these quantities could also be found in the ITER physics basis document (ITER Physics Basis, 1999). Finally, table 2 provides an overview of the range of some plasma parameters selected for this analysis together with the corresponding values expected in ITER scenarios according to Green et al (2003).

\section{Operational domain of advanced tokamak regimes}

This section, devoted to the assessment of the advanced tokamak regimes using data from the multi-machine international database, is divided in three main parts. In section 3.1, the performances are studied from the perspective of possible steady-state operation, then the operational limits are discussed in section 3.2. Finally, in section 3.3 the operational domain of the advanced regimes is mapped in terms of their dimensionless parameters such as $\rho^{*}, v_{\mathrm{e}}^{*}$ to assess quantitatively the gap between present day and next step tokamak experiments.

\subsection{Fusion performances for long pulse and steady-state operation}

The operational domain of advanced tokamak regimes achieved with monotonic or nonmonotonic $q$-profiles is first assessed, both in terms of their fusion performance and capability 
Table 2. Overview of the range of some plasma parameters selected for this analysis and the expected values for the inductive and non-inductive ITER operational regimes according to Green et al (2003). The data are from the tokamaks and regimes presented in table 1.

\begin{tabular}{llll}
\hline Parameters & $\begin{array}{l}\text { Range of data selected } \\
\text { in this paper }\end{array}$ & $\begin{array}{l}\text { ITER } Q \sim 10 \\
\text { inductive regime }\end{array}$ & $\begin{array}{l}\text { ITER } Q \sim 5 \\
\text { non-inductive regime }\end{array}$ \\
\hline$R[\mathrm{~m}] / a[\mathrm{~m}]$ & $0.87-3.5 / 0.23-1.0$ & $6.2 / 2.0$ & $6.35 / 1.85$ \\
$\kappa / \delta$ & $1.0-1.97 / 0-0.85$ & $1.7 / 0.33$ & $1.85 / 0.4$ \\
$B_{0}[\mathrm{~T}]$ & $1.4-5.4$ & 5.3 & 5.18 \\
$I_{\mathrm{p}}[\mathrm{MA}]$ & $0.072-3.6$ & 15 & 9 \\
$q_{95}$ & $2.8-15.8$ & 3 & $5.2-5.4$ \\
$n_{\mathrm{el}}\left[10^{19} \mathrm{~m}^{-3}\right]$ & $0.65-11.7$ & $10.1-12.3$ & $6.5-6.7$ \\
$\left\langle T_{\mathrm{e}}\right\rangle[\mathrm{keV}]$ & $0.5-7.7$ & $8.8-10.0$ & $10-12$ \\
$\tau_{E}[\mathrm{~s}]$ & $0.0024-1.07$ & $3.4-3.7$ & $3.1-2.5$ \\
$\tau_{\mathrm{D}}[\mathrm{s}]$ & $0.022-185$ & 400 & 3000 \\
$H_{89}$ & $\leqslant 3.7$ & 2 & $3-3.4$ \\
$\beta_{\mathrm{N}}$ & $\leqslant 3.9$ & $1.8-2.2$ & $2.8-3$ \\
$\beta_{\mathrm{p}}$ & $\leqslant 3.2$ & 0.6 & 1.5 \\
$\rho^{*}$ & $(1.6-24.3) \times 10^{-3}$ & $(1.8-2.2) \times 10^{-3}$ & $(2.1-2.6) \times 10^{-3}$ \\
$v_{\mathrm{e}}^{*}$ & $0.018-3.2$ & $0.030-0.04$ & $0.018-0.035$ \\
\hline
\end{tabular}

for steady-state. A machine-size independent figure of merit for optimizing the fusion power amplification factor, $Q$, and the fusion power density can be expressed as $\left(H_{\mathrm{ITER}-89 \mathrm{P}} \times \beta_{\mathrm{N}}\right) / q_{95}^{2}$ (e.g. Wolf (2003) for a justification of this parameter). It is expected that the non-inductive long pulse regimes in ITER should reach $\left(H_{\text {ITER-89P }} \times \beta_{\mathrm{N}}\right) / q_{95}^{2} \sim 0.35-0.45$ in the 'hybrid' scenario (at $q_{95} \sim 3-4$ ) and 0.3-0.4 in fully non-inductive current drive conditions at higher $q_{95} \sim 4-5$ for a fusion power amplification factor, $Q$, around 5 (Green et al 2003). The figure of merit of the plasma fusion performance, $\left(H_{\text {ITER-89P }} \times \beta_{\mathrm{N}}\right) / q_{95}^{2}$, is first plotted in figure $1(a)$ versus the poloidal beta times the square root of the inverse aspect ratio, $\varepsilon^{1 / 2} \beta_{\mathrm{p}}$. The fraction of self-generated bootstrap current to the total plasma current, $I_{\mathrm{boot}} / I_{\mathrm{p}}$, is proportional to $\varepsilon^{1 / 2} \beta_{\mathrm{p}}$. Efficient steady-state tokamak reactor operation will require optimizing the bootstrap current fraction approaching unity so as to reduce the external sources of non-inductive current. Therefore, high fusion performances for steady-state operation require to simultaneously increase $\left(H_{\text {ITER-89P }} \times \beta_{\mathrm{N}}\right) / q_{95}^{2}$ and obtain high values of $\varepsilon^{1 / 2} \beta_{\mathrm{p}}$. Data from ASDEX Upgrade, DIII-D, FT-U, JET, JT-60U, TCV and Tore Supra have been selected and the dataset has been classified according to the range of $q_{95}$ values. In addition, the filled symbols in figure 1( $a$ ) correspond to the performances sustained for a duration larger than 10 confinement times. This diagram could be interpreted by dividing it in three domains: (i) (the upper left corner) the high performance domain where a maximum value $\left(H_{\text {ITER-89P }} \times \beta_{\mathrm{N}}\right) / q_{95}^{2} \sim 0.8$ has been reached (ASDEX Upgrade, JT-60U) but that could not be sustained in the steady-state (low $\beta_{\mathrm{p}}$ ), (ii) (the lower right corner) the very high $\beta_{\mathrm{p}}$ regimes that are generally obtained at low plasma current and consequently at reduced fusion performances, (iii) (diagonal) an intermediate domain where high performances have been obtained at moderate $\beta_{\mathrm{p}}$ and at $q_{95} \sim 3.4-5$. For comparison, the expected operating domains for either $Q \sim 10$ inductive or $Q \sim 5$ noninductive operation in ITER are also indicated (according to Green et al (2003)). The expected domain of the steady-state $Q \sim 5$ non-inductive regime in ITER $\left(\beta_{\mathrm{p}} \sim 1.5, \varepsilon^{1 / 2} \beta_{\mathrm{p}} \sim 0.8\right)$ is lying in the so-called intermediate domain of operation. Figure $1(a)$ clearly indicates that $\left(H_{\text {ITER-89P }} \times \beta_{\mathrm{N}}\right) / q_{95}^{2} \sim 0.3-0.4$ at $\beta_{\mathrm{p}}$ exceeding unity $\left(\varepsilon^{1 / 2} \beta_{\mathrm{p}} \sim 0.55\right)$ have been reached and sustained for at least 10 confinement times at $q_{95} \sim 3.4-5$. This range of parameters is required for ensuring $Q \sim 5$ steady-state regime in ITER with typically $50 \%$ of bootstrap current. In addition, for a reduced number of discharges the figures of merit for fusion performances 

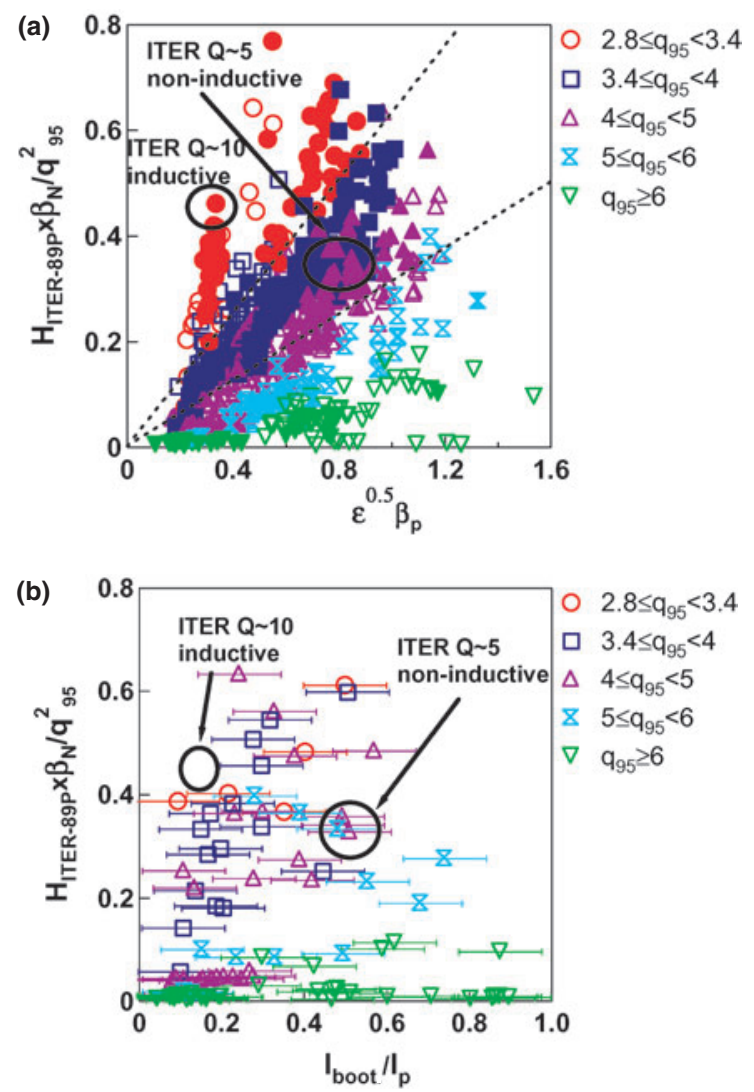

Figure 1. (a) Figures of merit of the plasma fusion performance, $\left(H_{\text {ITER- } 89 \mathrm{P}} \times \beta_{\mathrm{N}}\right) / q_{95}^{2}$, versus $\varepsilon^{1 / 2} \beta_{\mathrm{p}}$ at various $q_{95}$. The filled symbols correspond to discharges where the duration of the high performance phase, $\tau_{\mathrm{D}}$, normalized to the confinement time, $\tau_{\mathrm{D}} / \tau_{E}$, is above 10 (ASDEX Upgrade, DIII-D, FT-U, JET, JT-60U, TCV and Tore Supra). Each point corresponds to one discharge. $(b)$ Figures of merit of the plasma fusion performance, $\left(H_{\text {ITER }-89 \mathrm{P}} \times \beta_{\mathrm{N}}\right) / q_{95}^{2}$, versus the bootstrap current fraction, $I_{\text {boot }} / I_{\mathrm{p}}$ at various $q_{95}$ (99 discharges from ASDEX Upgrade, DIII-D, FT-U, JET, JT-60U, TCV and Tore Supra). Each point corresponds to one discharge.

have been directly plotted versus the actual bootstrap current fraction, $I_{\text {boot }} / I_{\mathrm{p}}$ as deduced from neoclassical calculation using the one-dimensional plasmas profiles (figure $1(b)$ ). This graph was motivated by the fact that the proportional factor between $I_{\mathrm{boot}} / I_{\mathrm{p}}$ and $\varepsilon^{1 / 2} \beta_{\mathrm{p}}$ is not constant and depends on the exact shape of plasmas profile (e.g. $q$-profile, existence of an ITB, etc). Therefore, the bootstrap current has been assessed using time dependent interpretative codes (ACCOME, ASTRA, CRONOS, TRANSP depending on the experiment) coupled to neoclassical modules that check the consistency of the various thermal profiles with the global integrated measurements (global energy content, neutron production, etc). Typically, $\left(H_{\text {ITER-89P }} \times \beta_{\mathrm{N}}\right) / q_{95}^{2} \sim 0.3-0.4$ has been obtained with $50 \%$ of bootstrap current. From this database, operation at higher bootstrap current fraction $(\sim 80 \%)$ as ultimately required for efficient steady-state tokamak reactor operation, is presently obtained at the expense of a reduction of fusion plasma performances.

The fusion plasma performances of each device, quantified by the (machine size dependent) triple fusion product, $n_{\mathrm{io}} T_{\mathrm{io}} \tau_{E}$, has also been plotted in figure 2 versus the duration of the high performance phase, $\tau_{\mathrm{D}}$, normalized to the confinement time, $\tau_{E}$. The triple fusion 


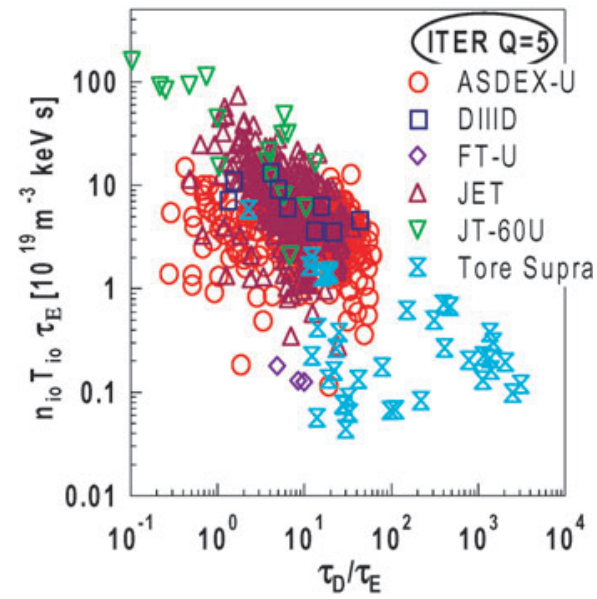

Figure 2. Fusion triple product, $n_{\mathrm{io}} T_{\mathrm{io}} \tau_{E}\left[10^{19} \mathrm{~m}^{-3} \mathrm{keV} \mathrm{s}\right]$ versus the duration of the high performance phase, $\tau_{\mathrm{D}}$, normalized to the confinement time, $\tau_{E}$. Each point corresponds to one discharge.

product is proportional to the fusion power density normalized to the plasma power losses. When evaluating the triple fusion product, $n_{\text {io }}$ and $T_{\text {io }}$ are, respectively, the core ion density (deuterium) and ion temperature. The central deuterium densities, $n_{\text {io }}$ have been calculated from the quasi-neutrality condition knowing the effective plasma charge, $Z_{\text {eff }}$, while assuming that carbon is the main impurity. For FT-U, $n_{\text {io }}$ has been deduced from impurity transport code that takes into account the highly ionized state of intrinsic metallic impurities such as Fe and Mo. The aim of this statistical analysis is more to evaluate the global trends rather than to discuss the exact value of a specific discharge. For reference, the expected operating domain for $Q \sim 5$ non-inductive operation in ITER is also indicated (according to Green et al (2003)). Figure 2 confirms the experimental difficulties in sustaining the highest fusion performance for long duration. A similar trend is observed in all the devices. This trend indicates that maintaining the plasma performances for a long duration requires operating the tokamak relatively far from its maximum operational limits (MHD limits, density limits, etc).

\subsection{Operational limits}

One of the important physical issues when extrapolating present day advanced tokamak regimes to ITER non-inductive scenarios is the possibility of sustaining improved confinement at 'high density'. 'High density' generally refers to densities approaching or exceeding the Greenwald density, $n_{\mathrm{G}}\left[n_{\mathrm{G}}=I_{\mathrm{p}} /\left(\pi a^{2}\right)\right.$ where the units are respectively, $\left.10^{20} \mathrm{~m}^{-3}, \mathrm{MA}, \mathrm{m}\right]$. In this context, the improved confinement factors obtained in ASDEX Upgrade, DIII-D, FTU, JET, JT-60U, TCV and Tore Supra experiments have been plotted in figure 3 directly versus: (i) the line averaged electron density, $n_{\mathrm{el}}$ (figure 3 (left)), (ii) the line averaged density normalized to the Greenwald density, $n_{\mathrm{el}} / n_{\mathrm{G}}$, (figure 3 (centre)) or (iii) the normalized collisionality, $v_{\mathrm{e}}^{*}$ figure 3 (right)) for various range of plasma triangularity. The expected operating domain for ITER $Q \sim 5$ non-inductive current drive scenario is also indicated (Green et al 2003). On the one hand, the 'high' confinement regimes $\left(H_{\text {ITER-89P }}>2.5\right)$ have generally been obtained at $n_{\mathrm{el}} / n_{\mathrm{G}}$ typically below $0.5-0.6$ (or at $n_{\mathrm{el}}$ below $(6-7) \times 10^{19} \mathrm{~m}^{-3}$ ). On the other hand, as it will be further discussed in section 3.3 , it is worth emphasizing that these data have been obtained at low normalized collisionality $\left(v_{\mathrm{e}}^{*}<0.03\right)$ approaching ITER collisionality regime 


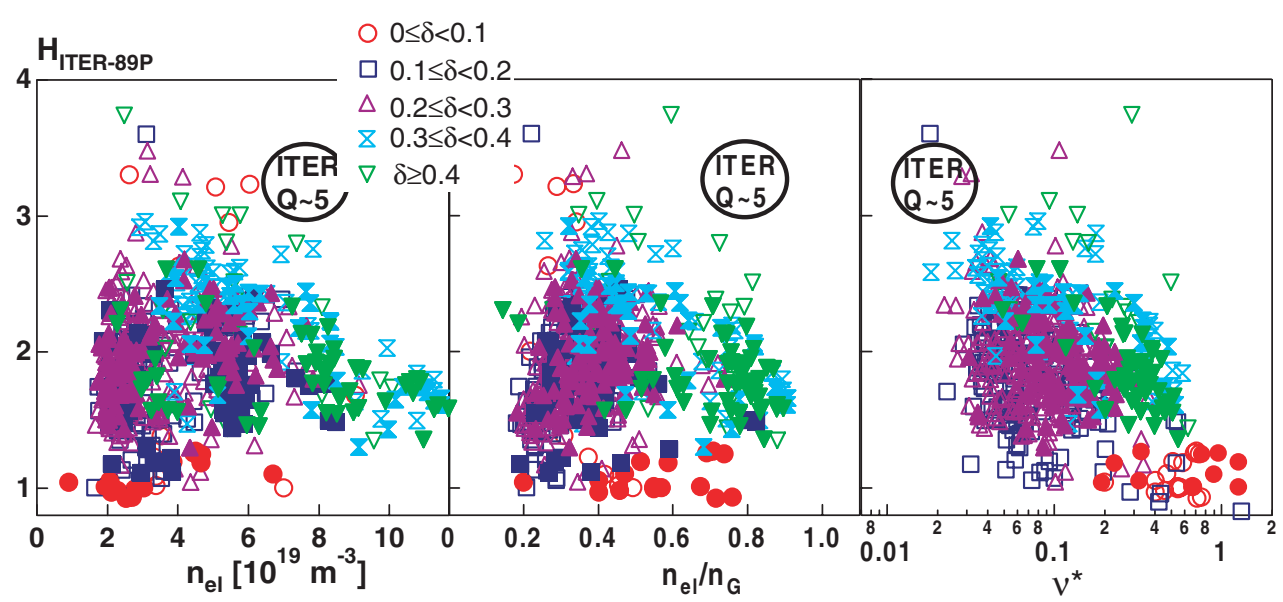

Figure 3. Improved confinement factor, $H_{\text {ITER- } 89 \mathrm{P}}$ versus (left) the line averaged density, $n_{\mathrm{el}}$ (centre) versus the line averaged density normalized to the Greenwald density, $n_{\mathrm{el}} / n_{\mathrm{G}}$, and (right) the normalized collisionality, $v_{\mathrm{e}}^{*}$, at various plasma triangularities, $\delta$. The filled symbols correspond to discharges where the duration of the high performance phase normalized to the confinement time, $\tau_{\mathrm{D}} / \tau_{E}$, is above 10 (ASDEX Upgrade, FT-U, DIII-D, JET, JT-60U, TCV and Tore Supra data). Each point corresponds to one discharge.

(figure 3 (right)). At $n_{\mathrm{el}} / n_{\mathrm{G}} \sim 0.8-1$ (or at $n_{\mathrm{el}}>7 \times 10^{19} \mathrm{~m}^{-3}$ ), improved confinement factors are mainly below 2. Finally, it should be stressed that as in the standard inductive H-mode regime, the magnetic configuration at high triangularity seems more favourable for reaching high density plasmas at high confinement. This common trend is probably related to the fact that the advanced regimes obtained at high triangularity combined the confinement properties of the edge transport barrier with a core improvement. Using data in the international database, the relative contribution between the edge and the core confinement enhancement could not be separated. Nevertheless, the difficulties in simultaneously reaching high density with a good core confinement in the advanced regimes could be understood as follows. When increasing the density, the flexibility to form and sustain the optimized current density profile for confinement and stability is reduced: (i) faster resistive inwards diffusion (lower temperature) of the transient off-axis Ohmic current induced during the current ramp-up phase of the discharge and (ii) lower level of non-inductively driven current by external means. Futhermore, the induced plasma rotation that has the potentiality to reduce the plasma turbulence through velocity shear is expected to be lower at higher density. Therefore, an important objective for future advanced tokamak experiments is to develop a route towards high confinement $\left(H_{\text {ITER-89P }} \sim 3\right)$ at high density (e.g. Ide et al (2002), Frigione et al (2003)).

Another major issue for the future of advanced tokamak regimes is to find an MHD stable route towards high normalized beta, $\beta_{\mathrm{N}}$, operation to ensure fusion performance together with a large fraction of self-generated bootstrap current. The onset of MHD instabilities is well described in terms of a critical normalized beta. This critical value depends on the plasma pressure and current density profiles. For instance, in the advanced regimes the $q$-profile is maintained above unity to avoid the $q=1$ seed island that may trigger neoclassical tearing mode instabilities that ultimately reduce the maximum achievable $\beta_{\mathrm{N}}$ values for long pulse operation. By shaping or controlling the current density profiles $\beta_{\mathrm{N}}$ values up to 3.9 have been obtained in DIII-D and ASDEX Upgrade, and of the order of 3 on JT-60U and JET. Figure 4 summarizes the performances achieved so far in terms of normalized beta in an operational diagram using the multi-machine zero-dimensional data. The $\beta_{\mathrm{N}}$ values of the 


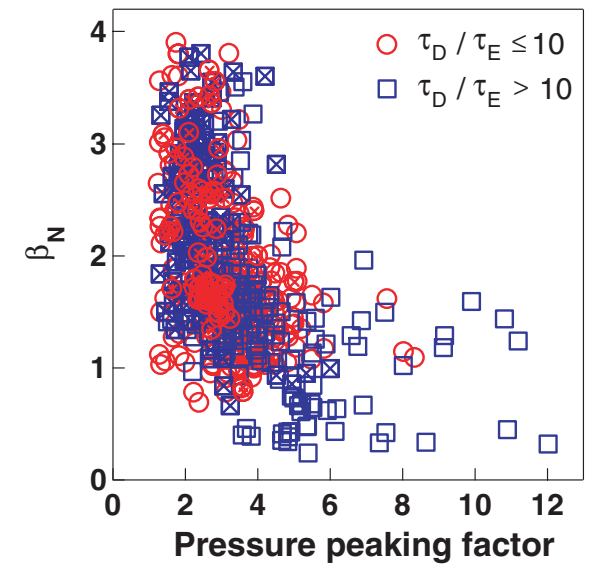

Figure 4. $\beta_{\mathrm{N}}$ versus the (electron) pressure peaking factor calculated as $\left(n_{\mathrm{eo}} \times T_{\mathrm{eo}}\right) /\left(n_{\mathrm{el}} \times\left\langle T_{\mathrm{e}}\right\rangle\right)$. Each point corresponds to one discharge. The symbols with a cross have been obtained in the 'hybrid' regime with monotonic $q$-profile ( $q$ on-axis $q_{0}=1-1.5$ and $q_{95} \leqslant 4.2$ ) (ASDEX Upgrade, FT-U, DIII-D, JET, JT-60U, TCV and Tore Supra data).

advanced regimes from ASDEX Upgrade, DIII-D, FT-U, JET, JT-60U, TCV and Tore Supra have been plotted versus a quantity representing the (electron) pressure peaking factor defined as $\left(n_{\mathrm{eo}} \times T_{\mathrm{eo}}\right) /\left(n_{\mathrm{el}} \times\left\langle T_{\mathrm{e}}\right\rangle\right)$. The open symbols with a cross indicate that these data have been obtained in the 'hybrid' regime with monotonic $q$-profile ( $q$ on-axis, $q_{0} \sim 1-1.5$ and $\left.q_{95} \leqslant 4.2\right)$. In addition, the $\beta_{\mathrm{N}}$ values have been classified according to the duration of the high performance phase of the discharges, with either $\tau_{\mathrm{D}} / \tau_{E} \leqslant 10$ or $\tau_{\mathrm{D}} / \tau_{E}>10$. It is worth mentioning that the number of discharges in this database with $\beta_{\mathrm{N}}>3$ and $\tau_{\mathrm{D}} / \tau_{E}>10$ (square symbols with a cross) is slightly more than four times higher in the hybrid regime compared to the other regimes. This might indicate the robustness of the hybrid scenario when reaching high $\beta_{\mathrm{N}}$ operation. Figure 4 shows that a broad pressure profile is favourable for raising $\beta_{\mathrm{N}}$. This is consistent with the ideal pressure driven kink modes that are destabilized with highly peaked pressure profiles generally obtained with improved core confinement plasmas. In order to broaden the pressure profiles for stability reasons, it is necessary to form and sustain wide ITBs (or improved core confinement region) at large plasma radius that encompass a wide region with reduced anomalous transport. To further broaden the pressure profile, improved core confinement is usually combined with an edge transport barrier (H-mode edge) (Lazarus et al 1996). Finally, it is worth pointing out that at a given value of the pressure peaking factor the spread over $\beta_{\mathrm{N}}$ is mainly due to the variation of the toroidal magnetic field, $B_{0}$. For instance, the highest values of $\beta_{\mathrm{N}}$ (typically $\beta_{\mathrm{N}} \geqslant 3$ ) are mainly obtained at reduced toroidal field (typically $B_{0} \leqslant 2.1 \mathrm{~T}$ ). This dependence will be revealed in figure 6 (left) of section 3.3, where $\beta_{\mathrm{N}}$ values are plotted versus the normalized radius, $\rho^{*}$, which takes into account the toroidal magnetic field dependence.

\subsection{Operation domain in terms of dimensionless parameters}

The existence domain of the present advanced mode of operation is also assessed using the dimensionless quantities such as the normalized Larmor radius, $\rho^{*}$, collisionality, $v_{\mathrm{e}}^{*}$, Mach number $M_{\Phi}$, and ratio of ion to electron temperature, $T_{\mathrm{i}} / T_{\mathrm{e}}$. These quantities govern the plasma physics properties, e.g. the neoclassical (bootstrap effect, etc) and anomalous plasma radial transport. The domain of plasma operation in the next step devices like ITER will be at values of $\rho^{*}, v_{\mathrm{e}}^{*}$ and Mach numbers, lower than the ones presently obtained, together 
with a ratio of $T_{\mathrm{i}} / T_{\mathrm{e}} \sim 1$. In ITER non-inductive current drive regime, it is expected to operate the plasmas with $\rho^{*} \sim 2 \times 10^{-3}$ and $v_{\mathrm{e}}^{*} \sim 2 \times 10^{-2}$. Plasmas with such low values of $\rho^{*}$ and $v_{\mathrm{e}}^{*}$ have not been obtained simultaneously in present day experiments. An open issue is therefore to assess the sensitivity of the plasma properties of the advanced regimes when varying these normalized plasma quantities. In this context, it is worth pointing out that similarity experiments between ASDEX Upgrade and JET in the 'hybrid' scenario have been recently performed at different $\rho^{*}$ values (Joffrin et al 2002, Sips et al 2003). To map the operation domain of present day advanced tokamak regimes in terms of these dimensionless parameters, the normalized collisionalities, $v_{\mathrm{e}}^{*}$, have been plotted versus the normalized Larmor radius, $\rho^{*}$ in figure 5 . The filled symbols in figure 5 correspond to high fusion performance discharges with the product $H_{\text {ITER-89P }} \times \beta_{\mathrm{N}}$ greater than 4 , i.e. above the standard inductive $\mathrm{H}$-mode regime performance. As in the previous plots, the expected ranges of $\nu_{\mathrm{e}}^{*}$ and $\rho^{*}$ values for the non-inductive current drive $Q \sim 5$ ITER scenario have been highlighted in this diagram. In addition, the plasma fusion performances quantified either by the normalized beta, $\beta_{\mathrm{N}}$ or the figure of merit $\left(H_{\text {ITER-89P }} \times \beta_{\mathrm{N}}\right) / q_{95}^{2}$ have been plotted versus $\rho^{*}$ for various ranges of the collisionality parameters (figure 6). Figures 5 and 6 indicate that operation at relatively low values of $\rho^{*}$ could be obtained at low powers (e.g. the Tore Supra data at high toroidal field, $B_{0} \sim 4 \mathrm{~T}$ ) but at high normalized collisionality, i.e. at low volume averaged temperature. On the other hand, low collisionality $\left(v_{\mathrm{e}}^{*} \leqslant 0.03\right)$ operation has been obtained at high temperature (e.g. the JT-60U data) but at $\rho^{*} \sim 5 \times 10^{-3}$. Interestingly enough, there is a domain of reduced collisionality and normalized Larmor radius $\left(v_{\mathrm{e}}^{*} \leqslant 0.05\right.$ and $\left.\rho^{*} \leqslant 5 \times 10^{-3}\right)$ approaching the required values for ITER that has been obtained on JET. This requires operating the JET device at high toroidal field $\left(B_{0} \geqslant 3.5 \mathrm{~T}\right)$, at high volume averaged temperature $\left(\left\langle T_{\mathrm{e}}\right\rangle \sim 3 \mathrm{keV}\right)$, moderate density $\left(\left\langle n_{\mathrm{e}}\right\rangle \sim(1.5-2) \times 10^{19} \mathrm{~m}^{-3}\right)$ and at $q_{95} \sim 4-5$ $\left(I_{\mathrm{p}} \sim 3 \mathrm{MA}\right)$. To further approach the ITER domain in the JET device would require increasing the applied power or/and the plasma volume for this range of parameters.

Another important issue when extrapolating the advanced regimes towards next step experiments, is the possibility of forming and sustaining these operation modes with low torque injection and with a ratio of ion to electron temperature around unity. In the context of an $\alpha$-particle heated plasmas, the fusion born fast $\alpha$-particles will predominantly heat the thermal electrons, which in turn will heat the ion species through collisional energy transfer resulting

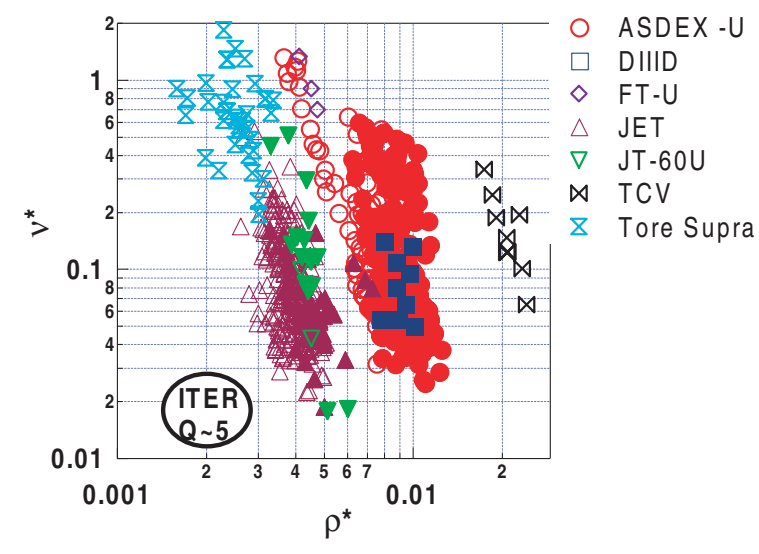

Figure 5. Operation domain of advanced tokamak regimes in terms of dimensionless parameters: normalized collisionality, $v_{\mathrm{e}}^{*}$, versus normalized Larmor radius, $\rho^{*}$. The filled symbols correspond to discharges where $H_{\text {ITER-89P }} \times \beta_{\mathrm{N}}>4$. Each point corresponds to one discharge. 

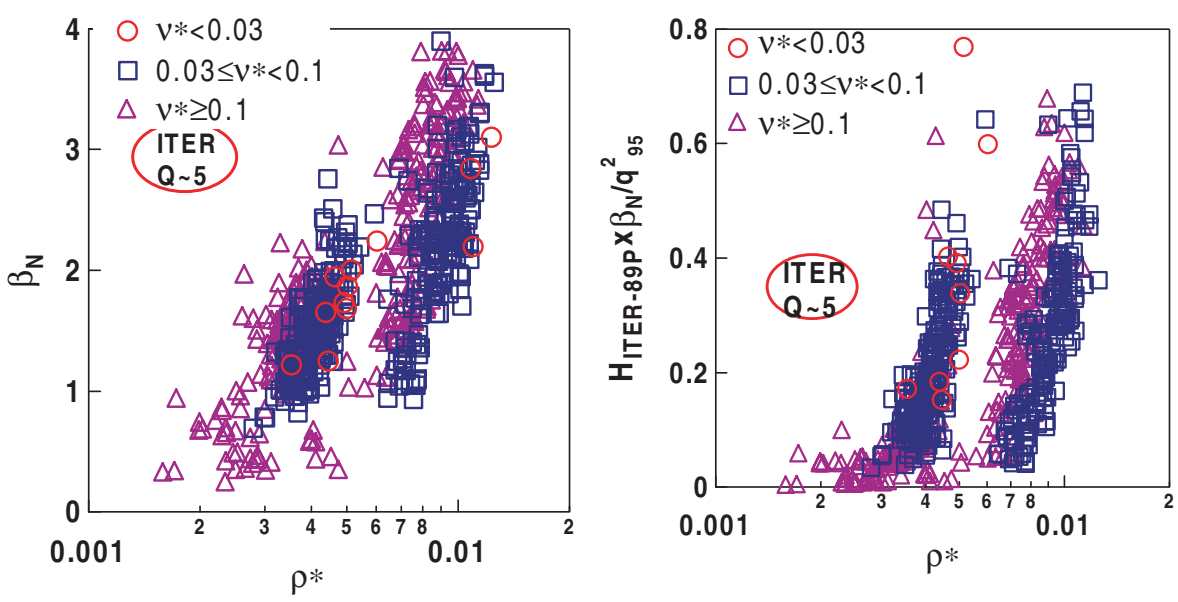

Figure 6. (Left) Normalized $\beta_{\mathrm{N}}$ and (right) figure of merit of the plasma performances, $\left(H_{\text {ITER-89P }} \times \beta_{\mathrm{N}}\right) / q_{95}^{2}$ versus the normalized Larmor radius for various collisionalities. Each point corresponds to one discharge (ASDEX Upgrade, FT-U, DIII-D, JET, JT-60U and Tore Supra data).

in a regime with $T_{\mathrm{e}} \sim T_{\mathrm{i}}$ at high plasma density where electron and ion are strongly coupled. In present day experiments, high performance regimes are generally obtained with dominant ion heating $\left(T_{\mathrm{i}}>T_{\mathrm{e}}\right)$ by injecting positive neutral beams that simultaneously combine core fuelling and momentum injection. To address this issue and to quantify the gap between present day experiments and next step operation, the performances measured by the normalized beta, $\beta_{\mathrm{N}}$ or the figure of merit $\left(H_{\text {ITER-89P }} \times \beta_{\mathrm{N}}\right) / q_{95}^{2}$ have been plotted versus the ratio of core ion to electron temperature $T_{\mathrm{io}} / T_{\mathrm{eo}}$ for various range of normalized plasma toroidal rotation or Mach number, $M_{\Phi}$ (figure 7). The absolute values of the core toroidal plasma rotation have been used to calculate the Mach numbers. Figure 7 (right) confirms that the highest figures of merit for fusion performances have been achieved for $T_{\mathrm{io}} / T_{\mathrm{eo}}>1$. Nevertheless, one should note that an interesting dataset exists that lies in region of parameters close to the ITER domain as far as the ratio of $T_{\mathrm{io}} / T_{\mathrm{eo}},\left(H_{\mathrm{ITER}-89 \mathrm{P}} \times \beta_{\mathrm{N}}\right) / q_{95}^{2}$ are concerned $\left(T_{\mathrm{io}} / T_{\mathrm{eo}} \sim 1\right.$ with $\left(H_{\text {ITER-89P }} \times \beta_{\mathrm{N}}\right) / q_{95}^{2} \sim 0.3-0.4$ and $\left.\beta_{\mathrm{N}}>2.5\right)$. These points correspond to experiments carried out on ASDEX Upgrade, JET and JT-60U. Finally, it is worth noting that these high performance regimes with $T_{\mathrm{io}} / T_{\mathrm{eo}} \sim 1$ have also been obtained at low Mach numbers, i.e. $M_{\Phi}<0.4$ (the red points in figure 7). On JT-60U an appropriate combination of co- and counter-current neutral beam injection has been used to operate with low applied torque. This illustrates that advanced regime could be achieved in condition of low torque injection as required for extrapolating these regimes to future reactor grade plasmas.

\section{Discussion and conclusion}

Using the global zero-dimensional data collected in the international multi-machine database, we have assessed and compared the plasma fusion performances of the advanced tokamak regimes achieved in ASDEX Upgrade, DIII-D, FT-U, JET, JT-60U, TCV and Tore Supra experiments. The results obtained in the present day tokamaks have been studied from the point of view of the required plasmas performances for achieving long pulses and ultimately steady-state full current drive operation in next step devices such as ITER. The extensive dataset on advanced tokamak operation selected in this paper consists of 753 discharges and for each discharge one time slice has been selected when the corresponding fusion yield reaches 

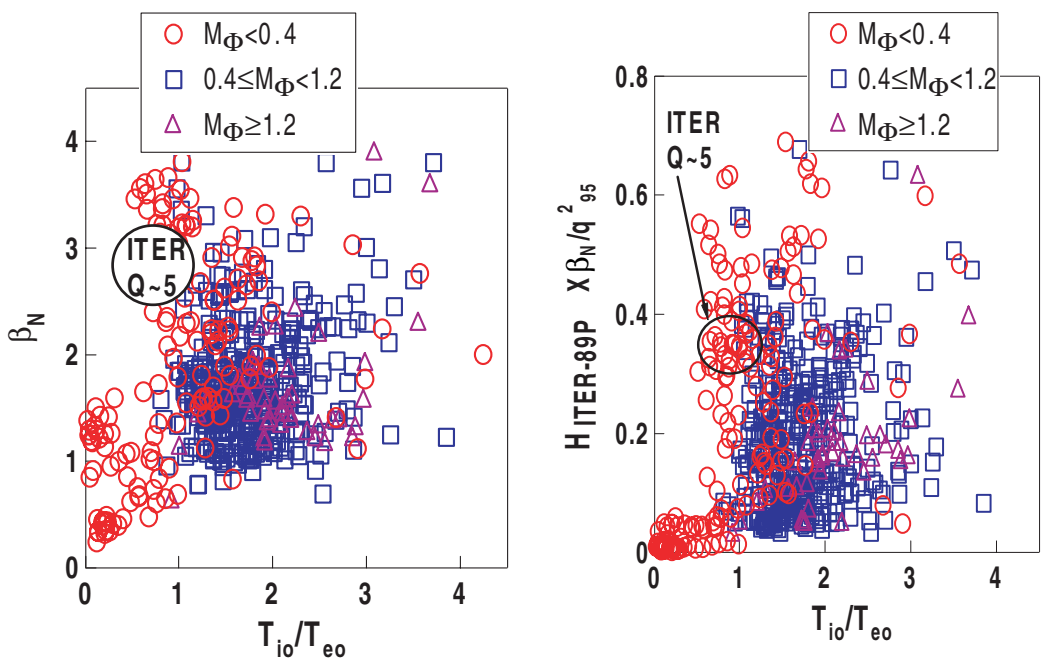

Figure 7. (Left) Normalized $\beta_{\mathrm{N}}$ and (right) figure of merit of the plasma performances, $\left(H_{\text {ITER-89P }} \times \beta_{\mathrm{N}}\right) / q_{95}^{2}$ versus the ratio of $T_{\mathrm{io}} / T_{\mathrm{eo}}$ for various absolute value of the Mach number, $M_{\Phi}$. Each point corresponds to one discharge (ASDEX Upgrade, FT-U, DIII-D, JET, JT-60U, TCV and Tore Supra data).

its maximum value. The performances towards steady-state operation, the operational limits (density and $\left.\beta_{\mathrm{N}}\right)$ and the plasma domains in terms of dimensionless parameters $\left(\rho^{*}, v_{\mathrm{e}}^{*}\right.$, etc) have been analysed using the international tokamak database.

A new operational diagram for advanced tokamak operation has been proposed where the dimensionless figure of merit, $\left(H_{\mathrm{ITER}-89 \mathrm{P}} \times \beta_{\mathrm{N}}\right) / q_{95}^{2}$, characterizing the fusion performance and confinement has been plotted versus the fraction of the plasma current driven by the bootstrap effect. The bootstrap current fraction has been either roughly quantified with the zerodimensional data in terms of $\varepsilon^{1 / 2} \beta_{\mathrm{p}}$ or for a reduced dataset (99 discharges) calculated with neoclassical codes using as inputs the various radial plasma profiles. In these diagrams, there is a continuous progression from the 'inductive' $\left(q_{95} \sim 3\right)$ to the 'hybrid' $\left(q_{95} \sim 3-4\right)$ and finally 'steady-state' $\left(q_{95} \sim 4-5\right)$ tokamak operating mode when simultaneously increasing the plasma performance together with the bootstrap current fraction. It turns out that advanced regimes have now reached figures of merit for performance, which are indeed those required for their extrapolation to the next step tokamak experiments, i.e. ITER. It has been reached with values simultaneously in the range of $\left(H_{\text {ITER-89P }} \times \beta_{\mathrm{N}}\right) / q_{95}^{2} \sim 0.4$ at $q_{95}<5$ and at a $\beta_{\mathrm{p}}$ exceeding unity for at least ten confinement times (typically with a bootstrap current fraction of 40-50\%). This range of plasma parameters is the one expected according to recent simulations for non-inductive current drive $Q \sim 5$ operation for ITER (Green et al 2003). Finally, it should be pointed out that operation at very high bootstrap current fraction $\left(I_{\text {boot }} / I_{\mathrm{p}} \sim 80 \%\right)$ that is ultimately required for efficient steady-state tokamak fusion reactor, is usually performed at reduced fusion performance and that the present set of experimental data is small in this operating space.

It has also been shown that fusion performances tend to decrease with the pulse duration: extending the plasma performances achieved on a short timescale usually requires operating the tokamak safely far from the operational limits. These limits are generally quantified in terms of the normalized toroidal beta, $\beta_{\mathrm{N}}$ and normalized density to the Greenwald density, $n_{\mathrm{el}} / n_{\mathrm{G}}$. As in standard inductive H-mode operation, the confinement enhancement factor in these advanced regimes tends to decrease when operating at high $n_{\mathrm{el}} / n_{\mathrm{G}}$. The highest values 
of both confinement enhancement and density have been mainly obtained at high triangularity $(\delta>0.4)$. So far, densities in the range of $n_{\mathrm{el}} / n_{\mathrm{G}} \sim 0.8\left(\right.$ or $\left.n_{\mathrm{el}}>(6-7) \times 10^{19} \mathrm{~m}^{-3}\right)$ have not been obtained at $H_{\text {ITER-89P }} \sim 3$ in advanced regimes, as required in non-inductive current drive operation of ITER. Further experimental effort in present day tokamaks should be devoted to investigating high confinement advanced tokamak regime at high density. High $\beta_{\mathrm{N}}$ operation in the range of 3 has been achieved with broad pressure profiles. The statistical analysis indicates that the width of the improved core confinement region (the region with reduced anomalous radial transport) should be large enough to avoid the development of a narrow domain with a too localized and very steep pressure gradient.

Finally, the operation domain of advanced regimes has been mapped in terms of dimensionless plasma parameters such as the normalized Larmor radius, $\rho^{*}$, collisionality, $\nu_{\mathrm{e}}^{*}$, Mach number, $M_{\Phi}$, and the ratio of ion to electron temperature, $T_{\mathrm{i}} / T_{\mathrm{e}}$. These parameters govern basic plasmas physics processes (e.g. neoclassical transport, bootstrap current, anomalous transport, etc). ITER plasmas will be in a very different domain of dimensionless plasmas parameters compared to regimes of present day tokamaks since it will require operating the plasmas with low values of $\rho^{*}, v_{\mathrm{e}}^{*}, M_{\Phi}$ and with $T_{\mathrm{i}} / T_{\mathrm{e}} \sim 1$. An important and open issue is to address how the present advanced regimes will extrapolate when the dimensionless plasmas parameters are varied. Advanced tokamak regimes allow operating present day devices at high confinement with core plasmas properties approaching normalized collisionalities, $v_{\mathrm{e}}^{*}$, as the ones expected in ITER. It has been recently shown that $\nu_{\mathrm{e}}^{*}$ is the correct dimensionless form of the plasma density (rather that $n_{\mathrm{el}} / n_{\mathrm{G}}$ ) when scaling confinement properties from present day tokamaks to larger one such as ITER (Petty et al 2004). Indeed, at the ITER collisionality $\left(v_{\mathrm{e}}^{*}<0.03\right)$, the figure of merit $\left(H_{\text {ITER-89P }} \times \beta_{\mathrm{N}}\right) / q_{95}^{2} \sim 0.3-0.4$ has been reached but at $\rho^{*} \sim(4-5) \times 10^{-3}$, i.e. two or three times above the expected values of $\rho^{*}$ for ITER $Q \sim 5$ non-inductive current drive regime. Matching simultaneously all the dimensionless parameters of present day experiment to the expected values in ITER could not be obtained. Nevertheless, cross-machine experiments where, for instance, the normalized Larmor radius is varied at fixed collisionality in non-inductive current drive conditions are indeed of major importance to check the relevance of the proposed regimes. The lowest values of $\rho^{*}$ and $\nu_{\mathrm{e}}^{*}$ (higher by a factor ranging between 2 and 3 with respect to the expected ITER values) at performance above the standard inductive tokamak operation $\left(H_{\text {ITER-89P }} \times \beta_{\mathrm{N}}>4\right)$ have been reached either in JT-60U or JET tokamaks that could be, in this context, considered as 'test-bed' for advanced regimes before extrapolating their performances to future larger tokamaks. In addition, these regimes should also be obtained and sustained with $T_{\mathrm{i}} / T_{\mathrm{e}} \sim 1$ at low Mach number (low torque injection). It has been shown that the highest plasma performances have now been reached for a ratio $T_{\mathrm{i}} / T_{\mathrm{e}}$ much larger than 1 . In this context, experimental effort should be devoted to developing high fusion yield regimes with a larger fraction of electron heating to operate at $T_{\mathrm{i}} / T_{\mathrm{e}}$ closer to unity while simultaneously keeping low values of $\rho^{*}$ and $v_{\mathrm{e}}^{*}$.

\section{Acknowledgments}

It is a great pleasure to acknowledge many fruitful discussions with Drs F Crisanti, D Moreau, A A Tuccillo and the members of the ITPA Group on Steady-State Operation and Energetic Particles.

\section{References}

Bécoulet A et al 2003 15th Topical Conf. on Radio Frequency Power in Plasmas (Moran, Wyoming, USA, 19-21 May 2003) 
Behn R et al 2003 Proc. 30th Eur. Conf. on Plasma Physics and Controlled Fusion (St Petersburg, 2003) vol 27A (Geneva: European Physical Society) P-3.208, http://eps2003.ioffe.ru/

Connor J W et al 2004 Nucl. Fusion at press

Campbell D J et al 2001 Phys. Plasmas 82041

Challis C D et al 2001 Plasma Phys. Control. Fusion 43861

Challis C D et al 2002 Plasma Phys. Control. Fusion 441031

Crisanti F et al 2002 Phys. Rev. Lett. 88 145004-1

Doyle E J et al 2001 Plasma Phys. Control. Fusion 43 A95

Equipe Tore Supra (presented by Litaudon X) 1996 Plasma Phys. Control. Fusion 38 A251

Fujita T et al 2001 Phys. Rev. Lett. 87085001

Fujita T et al 2002a Nucl. Fusion 42180

Fujita T et al 2002b Proc. 19th IAEA Fusion Energy Conf. (Lyon) (Vienna: IAEA) OV/1-3

Fujita T et al 2003 Proc. 30th Eur. Conf. on Plasma Physics and Controlled Fusion (St Petersburg, 2003) vol 27A (Geneva: European Physical Society) P-2.131, http://eps2003.ioffe.ru/

Fukuda T et al 2001 Proc. 28th Eur. Conf. on Plasma Physics and Controlled Fusion (Funchal, 2001) vol 25A (Geneva: European Physical Society) p 1349

Frigione D et al 2003 Proc. 30th Eur. Conf. on Plasma Physics and Controlled Fusion (St Petersburg, 2003) vol 27A (Geneva: European Physical Society) P-2.91, http://eps2003.ioffe.ru/

Géraud A et al 1994 Proc. 21st Eur. Conf. on Plasma Physics and Controlled Fusion and (Montpellier, 1994) vol 18B (part I) (Geneva: European Physical Society) p 298

Gohil P et al 2002 Plasma Phys. Control. Fusion 44 A37

Gohil P et al 2003 Nucl. Fusion 43708

Gormezano C 1999 Plasma Phys. Control. Fusion 41 B367

Green B J et al 2003 Plasma Phys. Control. Fusion 45687

Henderson M A et al 2003 Phys. Plasmas 101796

Hoang G T et al 2002 Proc. 29th Eur. Conf. on Plasma Physics and Controlled Fusion (Montreux, 2002) vol 26B (Geneva: European Physical Society) p P-4.068, http://crppwww.epfl.ch/eps2002/

Ide S et al 2002 Plasma Phys. Control. Fusion 44 L63

ITER Physics Basis 1999 Nucl. Fusion 392203

Jacquinot J et al 2002 Recent development in Cadarache towards steady-state operation Proc. 19th IAEA Fusion Energy Conf. (Lyon, France) (Vienna: IAEA)

JET Team (prepared by C Gormezano) 1999 Nucl. Fusion 391875

Joffrin E et al 2002 Plasma Phys. Control. Fusion 441203

Kaye S M et al 1997 Nucl. Fusion 371303

Kikuchi M 1990 Nucl. Fusion 30265

Kamada Y et al 2001 Nucl. Fusion 411311

Lazarus E A et al 1996 Phys. Rev. Lett. 772714

Litaudon X 1998 Plasma Phys. Control. Fusion 40 A251

Litaudon X et al 2002 Plasma Phys. Control. Fusion 441057

Litaudon X et al 2003 Nucl. Fusion 43565

Luce T C et al 2003 Nucl. Fusion 43321

Pericoli-Ridolfini V et al 2003 Nucl. Fusion 43469

Petty C C, Luce T C, Cordey J G and McDonald D C 2004 Plasma Phys. Control. Fusion 46 A207

Saoutic B et al 1994 Plasma Phys. Control. Fusion 36 B123

Sauter O et al 2002 Steady-state fully non-inductive reverse shear scenarios with electron ITB and dominant bootstrap current Proc. 19th IAEA Fusion Energy Conf. (Lyon, France) (Vienna: IAEA) p P5-06

Sips A C C et al 2002a Plasma Phys. Control. Fusion 44 A391

Sips A C C et al 2002b Plasma Phys. Control. Fusion 44 B69

Sips A C C et al 2003 Proc. 30th Eur. Conf. on Plasma Physics and Controlled Fusion (St Petersburg, 2003) vol 27A (Geneva: European Physical Society) O1-3A, http://eps2003.ioffe.ru/

Strait E J et al 1995 Phys. Rev. Lett. 754421

Taylor T S 1997 Plasma Phys. Control. Fusion 39 B47

Wade M R et al 2003 Nucl. Fusion 43634

Wolf R C et al 1999 Plasma Phys. Control. Fusion 41 B93

Wolf R C et al 2001 Nucl. Fusion 411259

Wolf R C 2003 Plasma Phys. Control. Fusion 45 R1 